\section{DENTISTS REBEL AGAINST REGULATOR}

On 5 December dentists from across the UK passed a motion of no confidence in their regulator, the General Dental Council (GDC).

Over 200 dentists and dental professionals from all around the country attended a special event at the Cavendish Conference Centre in London to ensure their voice was heard and to register their discontent at the actions of the GDC.

The motion that "this conference believes the GDC has failed in its role as the regulator for dentistry in its current model and demands a reformation of the GDC that will protect patients and re-establish the support of the dental profession' was passed unanimously.

Ian Gordon, spokesperson for the alliance of local dental committees (LDCs), said: 'Fundamental reform is clearly needed, starting with a return to mutual trust and away from a culture of fear. Proportional regulation led by a regulator who understands the profession is a prerequisite to progress'.

In response to the motion, the GDC said in a statement that 'it is disappointing that the LDCs have chosen to go down this route and it is of extreme concern to the GDC that they are construing this ARF increase as potentially

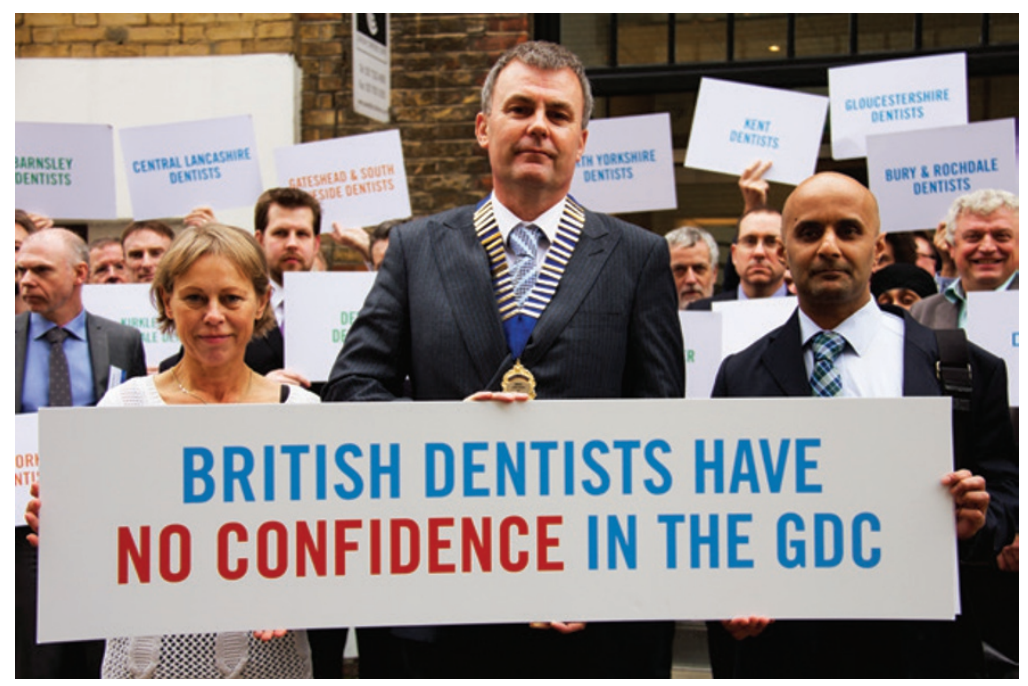

having an impact on patient access to dental services. This unfounded and inflammatory stance will do nothing but worry patients unnecessarily'.

MPs debated the GDC's performance on 9 December, six days before the GDC was scheduled to defend itself in the High Court. Editor's note: As this issue of the BDJ goes to press a verdict from the BDA vs GDC judicial review has not yet been announced. Justice Cranston was still considering the case as of 18 December 2014.

\section{BOOK REVIEW

$\begin{aligned} & \text { The Dental Press } \text { THE DENTAL } \\ & \text { PRESS } \\ & \text { N. Wilson, S. Gelbier } \\ & \text { BDA } \\ & \text { price } £ 6.95 ; \text { pp } 95 \\ & \text { BDinesssumus } \\ & \text { ISBN 9781291769166 }\end{aligned}$

This completes a current series of five transcripts recounting "witness' seminars held at the BDA.

The BDA Museum and the Unit for the History of Dentistry at King's College London Dental Institute facilitated the seminars which offered a platform for participants to discuss their memories and experiences of the dental profession since the NHS was established in 1948. Other seminar subjects range from regulation of the dental profession to dental materials to the subject of this transcript: the dental media.

Participants in this seminar, from September 2012, included editors of current dental periodicals, many of whom also have impressive clinical backgrounds.

The aim of these 'fly on the wall' transcripts is to allow the reader a unique insight into dentistry that could not otherwise be gained. It hopes to create an 'archive of a living history of dentistry' made possible by the late Dr John McLean OBE who left a generous bequest to fund this unique study of the profession.

The opening section details a tribute to Dr John McLean, and then the transcript begins. This is one single bulk of text spanning the remaining 84 pages. There are no chapters to break up the text. This unusual format allows the reader to feel they are a part of the events, but does not readily provide natural breaks to stop reading without losing track of the discussion.

The occasionally lively dialogue touches on the benefit that dental periodicals have had within the profession over the years, and the perceptions that the public may have developed about the dental profession as a whole via information from the commercial media.

It is difficult to pinpoint who the target audience of this production is. There is a nostalgic element for dentists who may recall some of the older publications. The subject matter may at first seem less than exciting, but some may find that this is redeemed by the unconventional transcript style which reads easily, as long as you have the time to read it in one sitting!

A. BROWNLIE

\section{WINTER LECTURE CONSIDERS FUTURISTIC FILLING MATERIALS}

The annual BDA/BDJ Winter Lecture took place on 10 December 2014 - the third such event to date. Introduced by $B D J$ Editor-in-Chief Stephen Hancocks OBE, this year Dr Michele Barbour (pictured) presented a lecture on 'Dental materials today and tomorrow'.

Dr Barbour is Senior Lecturer in Dental Biomaterials and the University of Bristol and head of the Oral

Nanoscience research group and Deputy

Head of Bristol Dental School. She serves on the editorial board of Caries Research and Journal of Dentistry, has published over 40 papers and over 60 conference abstracts. Recently BDJ Managing Editor Dr Ruth Doherty interviewed Dr Barbour for the BDJ $(2014 ; 217$ : 494-495).

In the lecture Dr Barbour discussed some new, and not-so-new, technologies available to today's dentists, and considered what really matters when it comes to dental restoratives. She then discussed what she considers to be the exciting directions that dental materials will take in the future.

The lecture was followed by a lively question and answer session and a festive reception. For those BDA members unable to attend, the lecture will be available to view via the BDA website and will attract one hour of CPD. 